\title{
SOBRE LA DENTALIZACIÓN DE -s- EN ESPAÑOL
}

\author{
Eugenio Martínez CELdRÁN \\ Universidad de Barcelona
}

En la Revista de Filología Española ${ }^{1}$ aparecieron dos artículos consecutivos sobre la dentalización de la -s- en español. El primero era mío y el segundo de A. Quilis, que manifestaba su intención de rebatir mis afirmaciones. Como en esa ocasión no tuve la oportunidad de examinar sus argumentos, paso ahora a hacerlo ${ }^{2}$.

- 1. En el primer punto de su escrito, después de citar a Navarro Tomás, el Dr. A. Quilis indica que «si [s] se dentaliza ante [t] o [d], o lo que es lo mismo, si una [s] es dental, su lugar de articulación, desde el principio hasta el final, es la cara interior de los incisivos superiores».

En primer lugar, Navarro Tomás y yo mismo hablamos de dentalización, no de $s$ dental, lo cual implica un proceso asimilatorio. Un proceso se concibe de forma dinámica $\mathrm{y}$, por tanto, no se puede exigir que «desde el principio hasta el final» permanezcan los órganos en el mismo lugar de articulación: bastará con que comience el proceso en alguna de sus fases: distensiva y/o tensiva. La anticipación es un tipo asimilatorio ${ }^{3}$.

\footnotetext{
${ }^{1}$ Tomo LXXV, 1995, fascículos $3 .^{\circ}$ y $4 .^{\circ}$, págs. 301 y sigs.

${ }^{2}$ Quisiera dejar claro dos cuestiones desde el principio: primera, quiero expresar mi respeto por el Dr. A. Quilis, que considero un maestro de la fonética española; segunda, todos los maestros, en la ciencia, han sido discutidos, lo cual, lejos de ser un demérito, es un reconocimiento de su trabajo y su labor, porque es señal de que se les presta gran atención.

3 Vid. «asimilación» en J. Dubois y otros, Diccionario de lingüística, Madrid, Alianza, 1973. Es importante subrayar el carácter dinámico de toda articulación: «Speech is a continuously changing acoustic stream produced by dinamic articulatory processes [...] Phonetics adaptations are variations in the ways in which articulators move and the extent to which cavities change shape, according to what phonemes are neighbors» (G. J. Borden y K. S. Harris, Speech Science Primer, Baltimore, Williams \& Wilkins, 1984, 2. ${ }^{\text {a }}$ edición, págs. 126-128). Merece la pena destacar este dinamismo para rechazar el concepto de que una asimilación forzosamente implica que el sonido «desde el principio hasta el final» sea de una determinada manera. Además, las autoras citadas distinguen entre adaptación y asimilación. Quizás el caso de la s dentalizada sea un caso de adaptación más que de asimilación en el sentido de estas autoras.
} 
En segundo lugar, cuando Navarro Tomás describe la articulación de [t], además de decir que «la punta de la lengua se apoya contra la cara interior de los incisivos superiores...», añade lo siguiente: «el contacto de la lengua se extiende más o menos, hacia arriba, por las encías y los alveolos» ${ }^{4}$. Según nuestros palatogramas, hay que eliminar lo de «más o menos», siempre hay contacto con los alveolos, cuestión que dejamos clara en nuestro artículo. Esto concuerda además con lo que dicen P. Ladefoged y I. Maddieson: «dental stops are usually laminal rather than apical, with contact on both the teeth and the front part of the alveolar ridge [...] the dental stops typically have a long contact region in the sagittal plane, and might better be regarded as laminal denti-alveolars rather than pure dentals» ${ }^{5}$. Estoy totalmente de acuerdo con estas afirmaciones de Ladefoged y Maddieson. En cambio, parece que A. Quilis defiende que las dentales sólo tienen contacto con «la cara interior de los incisivos superiores» ${ }^{6}$; es decir, son dentales puras. No parece que esto sea así ni en español, ni en las lenguas del mundo en general. Como prueba de ello, al final del artículo insertamos dos figuras, la primera representa la sucesión de palatogramas de la secuencia [áta] y, la segunda, de la secuencia [ána]; esas dos figuras muestran claramente la diferencia entre una dentoalveolar y una alveolar; como siempre hemos dicho, la dental cubre todas las filas de los alveolos mientras que la alveolar sólo cubre las posteriores y deja libre la primera.

2. Efectivamente, el instrumento utilizado es un electropalatógrafo y es cierto que no informa sobre la región dental, entendida ésta como «la cara interior de los incisivos superiores»; pero sí informa sobre los alveolos y, como se ha visto en el punto anterior, nuestras dentales son dentoalveolares y no dentales puras. La primera línea de electrodos de nuestros paladares artificiales está situada al principio de la zona alveolar: allí donde los dientes entran en contacto con la encía y esa primera línea es la que presenta diferencias significativas (estadísticamente determinadas) entre la [s] dentalizada y la alveolar pura.

\footnotetext{
${ }^{4}$ Manual de pronunciación española, Madrid, CSIC, 1971, decimosexta edición, pág. 96.

5 The Sounds of the World's Languages, Cambridge, Massachusetts, Blackwell Publishers, 1996, págs. 20 y 21.

${ }^{6}$ En la nota a pie de página núm. 11 del escrito del Dr. A. Quilis dice que «la oclusión del ápice lingual contra la cara interior de los incisivos para la pronunciación de [t] hace que, por la forma que adopta la lengua en su recorrido desde la [s] alveolar a la [t] dental, se establezca contacto también entre el predorso de la lengua y la región alveolar». Es decir, que para él el contacto de [t] con los alveolos es sólo un efecto de la coarticulación de la [s] alveolar. Por tanto, la dental es pura siempre, menos en estos casos esporádicos. Ya hemos visto que Navarro Tomás consideraba que la [t] tenía más o menos contacto siempre y que Ladefoged y Maddieson aseguran que en las lenguas del mundo esto es lo que sucede de forma general, lo cual quita la razón al Dr. A. Quilis.
} 
3. En el punto núm. 4 de su escrito, el Dr. A. Quilis rechaza mi interpretación de la figura 4B. Creo que es precisamente en ella donde mejor se refleja el proceso asimilatorio tal y como se ha definido anteriormente: las tramas 57-59 son semejantes a la de una [s] intervocálica, mientras que las tramas 60-65 son totalmente diferentes (3 frente a 6): invasión parcial, aunque amplia; no sólo se va cerrando la articulación sino que se van cubriendo todas las posiciones que implican el contacto dentoalveolar, como se aprecia en [t]; por tanto, la asimilación es total. La figura $4 \mathrm{~B}$ se corresponde con $4 \mathrm{~A}$ y $4 \mathrm{C}$, no hay que olvidarlo, porque en la figura 5 se pronuncia una $s$ dental intervocálica [ása] ${ }^{7}$, consciente de que este sonido no aparece en español en ese contexto $\mathrm{y}$, como se ve, la figura 5 tiene mayor semejanza con $4 \mathrm{C}$ [ásta], que con 3 [ása]. Por tanto, no es cierto que la progresión de contactos se deba a la oclusión de [t] de forma exclusiva, sino que demuestra que hay un avance de la lengua en [s] dentalizada.

4. En el punto 6, el Dr. Quilis afirma taxativamente que «si la constricción es alveolar, la consonante resultante es estridente, y si es dental, la consonante es mate». No sé cuál es la fuente de esta afirmación, pero no concuerda en absoluto con lo que se sabe en fonética general. P. Ladefoged y I. Maddieson, por ejemplo, en el apartado 5.2. titulado Sibilants, indican que "the more usual fricatives in the dental and alveolar regions are the sibilant fricatives $\mathbf{s}_{n}, \mathrm{z}, \mathrm{s}, \mathrm{z} . »$ Más adelante afirman incluso que «it is also possible to produce sibilant fricatives with a dental constriction, in the same region as that used for the non-sibilant $[\theta]$ sounds» ${ }^{8}$. Como se sabe, las sibilantes son fricativas estridentes en todos los casos ${ }^{9}$. Por tanto, la dentalización no tiene por qué impedir que su realización siga siendo estridente o sibilante. De ahí que en los sonogramas no se vea un cambio significativo en la dentalización. Navarro Tomás, al hablar de la dentalización de [s],

\footnotetext{
7 En mi artículo anterior hubo un descuido al no poner la referencia a la figura 5 en la pág. 303, al final del quinto párrafo, y al no aclarar en el pie de esa figura que correspondía a una [ș] dental intervocálica. De hecho, yo hice inconscientemente una distinción clara entre s dentalizada y s dental, por eso no indiqué que era intervocálica.

8 Op. cit., pág. 145. El subrayado dental es mío.

${ }^{9}$ Así lo indican, por ejemplo, R. Jakobson, G. Fant y M. Halle en Preliminaries to speech analysis: The distinctive features and their correlates, Cambridge, Massachusetts, The MIT Press, 1952, pág. 24: son estridentes «the hissing and hushing sibilants». Además dicen: "A supplementary barrier that offers greater resistance to the air stream is necessary in the case of the stridents [...] the sibilants employ the lower teeth...» (el subrayado es mío). Es decir los dientes inferiores sirven de barrera para producir la estridencia en todas las sibilantes. Por tanto, el Dr. A. Quilis no lleva razón cuando dice que la consonante dental es mate "porque no hay barrera suplementaria después de la constricción dental» (nota 16 de su escrito): olvida que esa barrera la constituyen los dientes inferiores «lower»; la traducción francesa de esta parte dice «les sifflantes utilisent les dents $d u$ dessous» (en R. Jakobson y L. Waugh, La charpente phonique du langage, Paris, Les Éditions de Minuit, 1980, pág. 172). El subrayado es mío.
} 
tampoco indica que haya un cambio en la sibilancia de esta consonante. Se limita a indicar un ligero cambio de punto de articulación exclusivamente y creo firmemente que, de haber pensado que existía algún cambio en la sibilancia, el maestro lo hubiera consignado fidedignamente.

5. No hemos puesto en duda la seriedad del viejo trabajo del Dr. A. Quilis por el hecho de afirmar que un único informante no es suficiente. Es sólo una cuestión estadística: cuanto mayor sea el número de informantes, menor probabilidad habrá de cometer error. Además, cualquier libro de estadística aconseja: «great care must be exercised in selecting samples if generalisation to the population is to be valid. Statistical methods of inferring the properties of populations from those of samples are based on the assumption that the samples are random» ${ }^{10}$. ¿Cómo puede ser la muestra aleatoria cuando se trata de un solo individuo?

\section{CONCLUSIÓN}

Me reafirmo en la creencia de que «todo el convencimiento de Quilis se ha basado sobre todo en el análisis acústico a partir de los sonogramas». Pero ahora he visto claro, y no llegué a darme cuenta de ello anteriormente, dónde se produce la principal diferencia de opiniones: para él una dental no puede tener nunca un timbre estridente (o sibilante) ${ }^{11}$; pero, como hemos visto en las afirmaciones de uno de los maestros de la fonética general - $\mathrm{P}$. Ladefoged-, no sólo existen dentales estridentes, sino que son las más habituales en las lenguas del mundo. Por tanto, no tiene por qué haber cambio en el modo de articulación en la dentalización de la $s$ española. Efectivamente, en los sonogramas no se aprecia ninguna diferencia significativa, lo cual no impide que pueda existir un cambio en el punto de articulación de ese sonido, como demuestra la relación cuántica existente entre el parámetro acústico y el articulatorio, tal como exponíamos en nuestro artículo anterior ${ }^{12}$.

Otra diferencia clave se sitúa en el uso de los términos dentalización y $s$ dental, que en el fondo encierran planteamientos divergentes. Navarro Tomás siempre utiliza dentalización. A lo largo de mi artículo, utilizo siempre dentalización y sólo hablo de $s$ dental para la pronunciada intervocálicamente, porque en ese caso no existe ningún proceso asimilatorio. El Dr. A. Quilis, por

\footnotetext{
${ }^{10}$ Ch. Butler, Statistics in Linguistics, Oxford, Basil Blackwell, 1985, pág. 2.

11 Por eso mismo él indicaba que «si predominase la constricción dental, las características acústicas serían bien diferentes, percibiendo en este caso un sonido próximo a $[\theta] »$ («Sobre los alófonos dentales de /s/», RFE, XLIX, 1966, pág. 343). Evidentemente, la dentalización no implica ningún sonido próximo a $[\theta]$.

${ }_{12}$ Por cierto, las leyendas de los ejes del gráfico 8 de mi artículo están intercambiadas: parámetro articulatorio debe estar en la abscisa y parámetro acústico en la ordenada.
} 
el contrario, sólo usa el término $s$ dental. Pienso que la diferencia entre ambos usos se sitúa en una concepción más dinámica y no discreta del primero, frente a una concepción más estática y discreta del segundo; esta última ha sido modificada por la teoría de la coarticulación ${ }^{13}$ en la fonética moderna.

FIGURAS
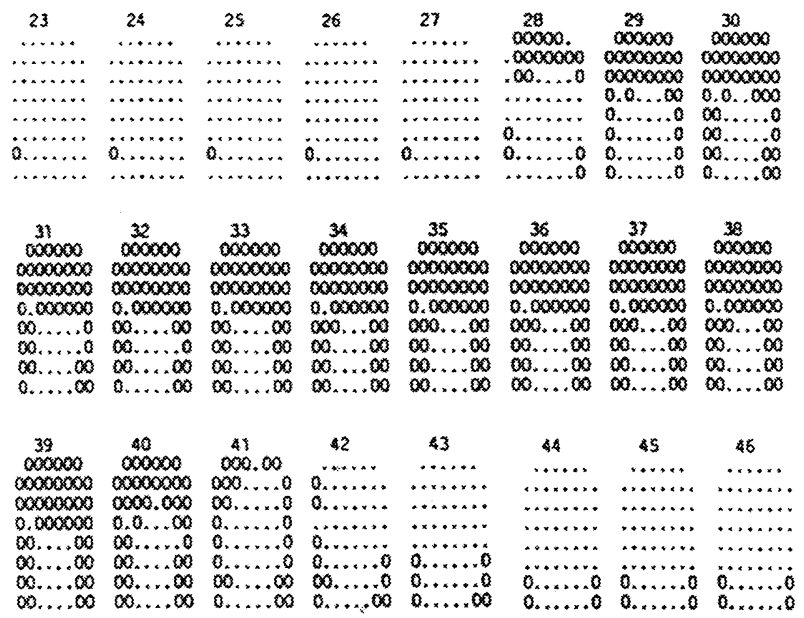

Figura 1: [áta], obsérvese el amplio contacto alveolar de [t] (tramas 28-41).
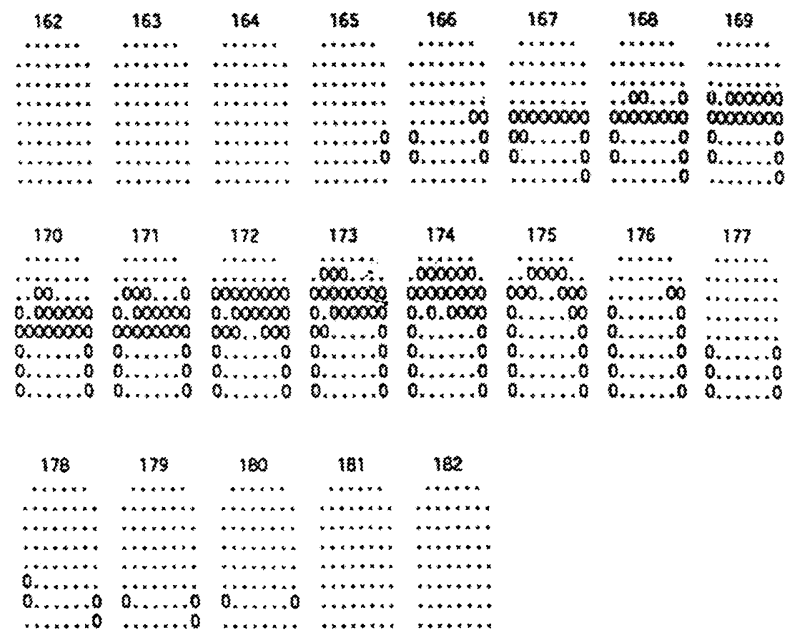

Figura 2: [ána], se deja sin contacto la primera fila (tramas 167-175).

${ }^{13}$ Cfr. M. A. Beckman, «Teoría fonética», en F. J. Newmeyer (ed.), Panorama de la Lingüistica Moderna, vol. I, Madrid, Visor, 1990, págs. 272 y sigs. 